\title{
On universality for linear combinations of L-functions
}

\author{
Takashi Nakamura • Lukasz Pańkowski
}

Received: 1 July 2010 / Accepted: 21 January 2011 / Published online: 15 February 2011

(C) The Author(s) 2011. This article is published with open access at Springerlink.com

\begin{abstract}
In the present paper, we consider the universality property in the Voronin sense for certain combinations of L-functions with general Dirichlet series as coefficients. In addition, we present some interesting examples of zeta and L-functions which can be expressed in this form. More precisely, we obtain the universality theorem for zeta functions associated to certain arithmetic functions, zeta functions associated to symmetric matrices and Euler-Zagier double zeta and L-functions.
\end{abstract}

Keywords Hybrid universality · Linear combination of L-functions · Zeros of L-functions - Zeta functions associated to arithmetic functions .

Zeta functions associated to symmetric matrices $\cdot$ Euler-Zagier double zeta-function

Mathematics Subject Classification (2000) $\quad 11 \mathrm{M} 32 \cdot 11 \mathrm{M} 35 \cdot 11 \mathrm{M} 41$

Takashi Nakamura was partially supported by JSPS Grants 21740024. Łukasz Pańkowski was partially supported by the Grant no. N N201 148233 from the Polish Ministry of Science and Higher Education.

T. Nakamura

Department of Mathematics, Faculty of Science and Technology,

Tokyo University of Science Noda, Chiba 278-8510 Japan

e-mail: nakamura_takashi@ma.noda.tus.ac.jp

Ł. Pańkowski ( $\varangle)$

Faculty of Mathematics and Computer Science,

Adam Mickiewicz University, Umultowska 87,

61-614 Poznan, Poland

e-mail: 1pan@amu.edu.pl 


\section{Introduction}

In 1975 Voronin [21,22] discovered a universality property of the famous Riemann zeta-function. More precisely, the modern version of the Voronin theorem can be formulated as follows.

Theorem A Let $K$ be a compact subset of the strip $D:=\left\{s \in \mathbb{C}: \frac{1}{2}<\operatorname{Re}(s)<1\right\}$ with connected complement and let a function $f(s)$ be non-vanishing and continuous on $K$ and analytic in the interior of $K$. Then, for every $\varepsilon>0$, we have

$$
\liminf _{T \rightarrow \infty} \frac{1}{T} \mu\left\{\tau \in[0, T]: \max _{s \in K}|\zeta(s+i \tau)-f(s)|<\varepsilon\right\}>0,
$$

where $\mu$ denotes the Lebesgue measure.

For the last 30 years numerous analogues of the Voronin theorem for other L-functions important in number theory were discovered by many mathematicians. For instance, universality theorems were proved for Dirichlet L-functions, Hecke L-functions, L-functions associated to newforms and many other. Furthermore, it turns out that there exist also a lot of zeta-functions with strong universality property, where the attribute strong means that also functions having zeros can be approximated. The first example comes from Bagchi [2] and Gonek [4], who showed, independently, the strong universality theorem for the Hurwitz zeta-functions $\zeta(s, \alpha)$, if $\alpha$ is transcendental or a rational number $\neq 1 / 2,1$. Afterwards, the strong universality was proved for many interesting generalizations of Hurwitz zeta-functions like Lerch zeta-functions (see $[12,15]$ ) or periodic Hurwitz zeta-functions (see [5,6]). For more examples and a comprehensive overview of the theory of universal zeta-functions we refer to the monograph [20].

A quite interesting and important extension of the Voronin universality theorem is due to Gonek (see Theorem A in [19]) and Kaczorowski, Kulas (see Theorem 3 in [7]). Recently, the second author (see [17,18]) improved this result and showed the so-called hybrid joint universality theorem.

Definition 1 We say that a set of functions $\left\{L_{1}, \ldots, L_{n}\right\}$ is hybridly jointly universal if for every compact set $K \subset D$ with connected complement, any functions $f_{1}, \ldots, f_{n}$ continuous and non-vanishing on $K$, which are analytic in the interior of $K$, any real numbers $\left(\alpha_{i}\right)_{(1 \leq i \leq m)}$ linearly independent over $\mathbb{Q}$ and any real numbers $\left(\theta_{i}\right)_{1 \leq i \leq m}$ we have

$\forall_{\varepsilon>0} \liminf _{T \rightarrow \infty} \frac{1}{T} \mu\left\{\tau \in[0, T]:\left\{\begin{array}{l}\max _{1 \leq j \leq n} \max _{s \in K}\left|L_{j}(s+i \tau)-f_{j}(s)\right|<\varepsilon \\ \max _{1 \leq j \leq m}\left\|\tau \alpha_{j}-\theta_{j}\right\|<\varepsilon\end{array}\right\}\right\}>0$,

where $\|\cdot\|$ denotes the distance to the nearest integer.

Moreover, we say that the set $\left\{L_{1}, \ldots, L_{n}\right\}$ is hybridly strongly jointly universal if functions having zeros can be approximated as well.

In [17] the second author proved hybrid joint universality for an axiomatically defined wide class of L-functions having Euler products, which contains for instance 
Dirichlet L-functions. Furthermore in [18] a strong hybrid joint universality theorem was proved for some L-functions without Euler product including Lerch zeta-functions, twists of Lerch zeta-functions and periodic Hurwitz zeta-functions.

In this paper we present some applications of a hybrid joint universality theorem. Mainly, we focus on certain linear combinations of L-functions with general Dirichlet series as coefficients. Recall that a general Dirichlet series is an arbitrary series of the form

$$
\sum_{n=1}^{\infty} a_{n} e^{-\lambda_{n} s}, \quad \text { where } a_{n} \in \mathbb{C}, \lambda_{n} \in \mathbb{R}
$$

Firstly, we prove that multiplying and adding absolutely convergent general Dirichlet series does not spoil the joint universality property.

Theorem 1 Suppose that the set $\left\{L_{1}, \ldots, L_{n}\right\}$ is hybridly jointly universal and $P_{1}, \ldots, P_{n}, Q_{1}, \ldots, Q_{n}$ are not identically vanishing general Dirichlet series, which are absolute convergent in the half-plane $\sigma>\frac{1}{2}$. Moreover, assume that the series $P_{j}$ are non-vanishing in the strip $D$. Then for $F_{j}(s)=P_{j}(s) L_{j}(s)+Q_{j}(s)$ a set $\left\{F_{1}, \ldots, F_{n}\right\}$ is strongly jointly universal in $D$ which means that for any compact set $K \subset D$ with connected complement, any functions $f_{1}, \ldots, f_{n}$ continuous on $K$ and analytic in the interior, satisfying $f_{j}(s) \neq Q_{j}(s)$ for all $s \in K$, we have

$$
\forall_{\varepsilon>0} \liminf _{T \rightarrow \infty} \frac{1}{T} \mu\left\{\tau \in[0, T]: \max _{1 \leq j \leq n} \max _{s \in K}\left|F_{j}(s+i \tau)-f_{j}(s)\right|<\varepsilon\right\}>0 .
$$

Moreover, if the set $\left\{L_{1}, \ldots, L_{n}\right\}$ is hybridly strongly jointly universal then the assumption that $f_{j}(s) \neq Q_{j}(s)$ for all $s \in K$ can be relaxed.

Note that the assumption that the general Dirichlet series are non-vanishing can not be relaxed. To show that it suffices to consider a function

$$
L(s)=\left(1-\frac{\sqrt[4]{2^{3}}}{2^{s}}\right) \zeta(s)
$$

which vanishes at points $\frac{3}{4}+2 k \pi i, k \in \mathbb{Z}$. Thus, for every compact set $K$ containing at least two zeros of this kind, there is no $\tau$ such that $\max _{s \in K}|L(s+i \tau)-1|<\frac{1}{2}$.

As usual, by Theorem 1 one can conclude the joint value approximation in the complex plane despite the assumption $f_{j}(s) \neq Q_{j}(s)$.

Corollary 1 We use the same notations and assumptions as in Theorem 1 . Let $1 / 2<$ $\sigma_{*}<1$ and $C_{k j}$ be arbitrary complex numbers for $0 \leq k \leq l$ and $1 \leq j \leq n$. Then for any $\varepsilon>0$, it holds that

$$
\liminf _{T \rightarrow \infty} \frac{1}{T} \mu\left\{\tau \in[0, T]: \max _{0 \leq k \leq l} \max _{1 \leq j \leq n}\left|F_{j}^{(k)}\left(\sigma_{*}+i \tau\right)-C_{k j}\right|<\varepsilon\right\}>0 .
$$


Proof Without loss of generality we can assume that $C_{0 j} \neq Q(0)$ for all $1 \leq j \leq n$, otherwise it suffices to take $C_{0 j}+\varepsilon$ instead of $C_{0 j}$. Moreover, for simplicity, suppose $n=1$. By [20, p. 194], we have that for any vector $\left(a_{0}, a_{1}, \ldots, a_{l}\right) \in \mathbb{C}^{l+1}$ with $a_{0} \neq 0$, there exists another vector $\left(b_{0}, b_{1}, \ldots, b_{l}\right) \in \mathbb{C}^{l+1}$ for which

$$
g(s):=\exp \left(\sum_{k=0}^{l} b_{k} s^{k}\right) \equiv \sum_{k=0}^{l} \frac{a_{k}}{k !} s^{k} \bmod s^{l+1} .
$$

Obviously, $h(s):=g(s)+Q(s) \neq Q(s)$ for all $s \in K$. By using Cauchy's formula and Theorem 1 and modifying the method in [20, p. 195], we have

$$
\left|F^{(k)}\left(\sigma_{*}+i \tau\right)-a_{k}-q_{k}\right|=\left|F^{(k)}\left(\sigma_{*}+i \tau\right)-h^{(k)}(0)\right|<\varepsilon,
$$

where $q_{k}:=Q^{(k)}(0)$. Hence the assertion follows.

Next, we deal with strong universality of linear combinations of L-functions with at least two terms.

Theorem 2 Suppose that a set $\left\{L_{1}, \ldots, L_{n}\right\}$ is hybridly jointly universal and $P_{1}, \ldots, P_{n}$ are not identically vanishing general Dirichlet series, which are absolute convergent in the half-plane $\sigma>\frac{1}{2}$. Moreover, assume that at least two of the series $P_{j}$ are non-vanishing in the strip $D$. Then, for $n \geq 2$, the function

$$
L(s)=\sum_{j=1}^{n} P_{j}(s) L_{j}(s)
$$

is strong universal in $D$, which means that for a compact set $K \subset D$ with connected complement, any function $f(s)$, continuous on $K$, which is analytic in the interior of $K$, and every $\varepsilon>0$, we have

$$
\liminf _{T \rightarrow \infty} \frac{1}{T} \mu\left\{\tau \in[0, T]: \max _{s \in K}|L(s+i \tau)-f(s)|<\varepsilon\right\}>0 .
$$

An immediate consequence of the Rouche theorem is the fact that every strongly universal function has infinitely many zeros in the strip $D$. Particularly, if the assumptions of Theorem 2 hold, then for the function $L(s)=\sum_{j=1}^{n} P_{j}(s) L_{j}(s)$ and every $1 / 2<\sigma_{1}<\sigma_{2}<1$ we have

$$
N_{L}\left(\sigma_{1}, \sigma_{2}, T\right) \gg T, \quad(T \rightarrow \infty),
$$

where $N_{L}\left(\sigma_{1}, \sigma_{2}, T\right)$ counts all zeros $\rho=\beta+i \gamma$ in the region $\sigma_{1} \leq \beta \leq \sigma_{2},|\gamma| \leq T$.

In fact, the assumption that at least two of the series $P_{j}$ are non-vanishing in $D$ turns out to be superfluous. 
Theorem 3 Suppose that a set $\left\{L_{1}, \ldots, L_{n}\right\}$ is hybridly (strongly) jointly universal and $P_{1}, \ldots, P_{n}$ are not identically vanishing general Dirichlet series, which are absolute convergent in the half-plane $\sigma>\frac{1}{2}$. Then for every $1 / 2<\sigma_{1}<\sigma_{2}<1$

$$
N_{L}\left(\sigma_{1}, \sigma_{2}, T\right) \gg T
$$

where

$$
L(s)=\sum_{j=1}^{n} P_{j}(s) L_{j}(s) \quad \text { and } \quad n \geq 2
$$

In Sect. 2 we present the proof of the theorems. Next, we conclude the paper with presenting some examples of L-functions which can be written as a linear combination of L-functions. In Sect. 3 we introduce some zeta functions associated to arithmetic functions like the Estermann zeta-functions, for which the universality theorem was proved in [3] under some extra assumption (see (1) in Section 3 below). Using Theorem 2, we are able to relax this assumption and simplify the proof of universality of the Estermann zeta-functions.

In the last two sections we prove the universality theorem for zeta functions associated to symmetric matrices and some multiple zeta functions.

\section{Proofs of the Theorems}

Basically, the proofs rely on the following lemma and methods presented by J. Sander and J. Steuding in [19, Sect. 2].

Lemma 1 Let $P_{1}, \ldots, P_{n}$ be general Dirichlet series given in the form $P_{k}(s)=$ $\sum_{n=1}^{\infty} a_{k n} e^{-\lambda_{k n} s}$. Then, for every $\varepsilon>0$ and every compact set $K$ lying in the halfplane of absolute convergence, there exist $\delta>0, N \in \mathbb{Z}_{+}$and a finite set $J \subset$ $\{1,2, \ldots, n\} \times \mathbb{Z}_{+}$such that the numbers $\lambda_{j m},((j, m) \in J)$, are linearly independent over $\mathbb{Q}$ and, moreover, if

$$
\max _{(k, n) \in J}\left\|\frac{\tau \lambda_{k n}}{N}\right\|<\delta
$$

then

$$
\max _{1 \leq k \leq n} \max _{s \in K}\left|P_{k}(s+i \tau)-P_{k}(s)\right|<\varepsilon
$$

Proof Let us fix $\varepsilon>0$ and a compact set $K$. Then there exists a positive integer $M$ such that

$$
\max _{1 \leq k \leq n} \max _{s \in K} \sum_{n>M}\left|a_{k n}\right| e^{-\lambda_{k n} \operatorname{Re} s}<\varepsilon / 3 .
$$


Now let us take the set $J$ of indices such that $\left\{\lambda_{j m}:(j, m) \in J\right\}$ is the basis of a vector space over $\mathbb{Q}$ generated by all numbers $\lambda_{k n}$ with $n \leq M$. Moreover let $N$ be a positive integer such that all these numbers can be represented as a linear combination of the elements $\lambda_{j m} / N,((j, m) \in J)$, with integer coefficients. Then, for $s \in K$ and $1 \leq k \leq n$, we have

$$
\begin{aligned}
\left|P_{k}(s+i \tau)-P_{k}(s)\right| & \leq \sum_{n \leq M}\left|a_{k n}\right|\left|\left(e^{-\lambda_{k n}(s+i \tau)}-e^{-\lambda_{k n}(s)}\right)\right|+2 \sum_{n>M}\left|a_{k n}\right| e^{-\lambda_{k n} \operatorname{Re} s} \\
& \ll \max _{n \leq M}\left|e^{-i \tau \lambda_{k n}}-1\right|+2 \varepsilon / 3 \ll \max _{(j, m) \in J}\left\|\frac{\tau \lambda_{j m}}{N}\right\|+2 \varepsilon / 3 .
\end{aligned}
$$

Finally, the proof is completed by taking suitable $\delta>0$.

Proof of Theorem 1 Combining hybrid (strong) joint universality of functions $L_{1}, \ldots$, $L_{n}$ and Lemma 1 shows that

$\liminf _{T \rightarrow \infty} \frac{1}{T} \mu\left\{\tau \in[0, T]:\left\{\begin{array}{l}\max _{1 \leq j \leq n} \max _{s \in K}\left|L_{j}(s+i \tau)-g_{j}(s)\right|<\varepsilon \\ \max _{1 \leq j \leq n} \max _{s \in K}\left|P_{j}(s+i \tau)-P_{j}(s)\right|<\varepsilon \\ \max _{1 \leq j \leq n} \max _{s \in K}\left|Q_{j}(s+i \tau)-Q_{j}(s)\right|<\varepsilon\end{array}\right\}>0\right.$.

Hence $F_{j}(s)=P_{j}(s) L_{j}(s)+Q_{j}(s)$ can approximate every function which can be written as $P_{j}(s) g_{j}(s)+Q_{j}(s)$. Thus it is clear that the functions $F_{1}, \ldots, F_{n}$ are strongly jointly universal, whenever $L_{1}, \ldots, L_{n}$ are hybridly strongly jointly universal.

Proof of Theorem 2 Without loss of generality we can assume that the series $P_{1}, P_{2}$ are non-vanishing. Let $H=\max _{s \in K}|f(s)|$. Then using Theorem 1 gives

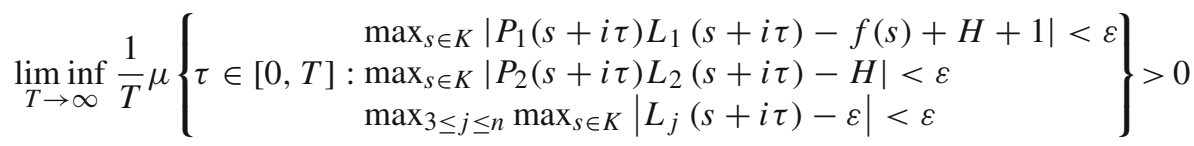

which completes the proof.

Proof of Theorem 3 Let us take $\sigma_{1}, \sigma_{2}$ such that $1 / 2<\sigma_{1}<\sigma_{2}<1$. Then there exist $\delta>0$ and $s_{0}=\sigma_{0}+i t_{0}$ such that $\sigma_{1}+\delta<\sigma_{0}<\sigma_{2}-\delta$ and $P_{j}(s)>0$ for $1 \leq j \leq n$, $s \in K=\left\{s \in \mathbb{C}:\left|s-s_{0}\right| \leq \delta\right\}$.

Arguing analogously to the previous proofs we obtain

$$
\liminf _{T \rightarrow \infty} \frac{1}{T} \mu\left\{\begin{array}{c}
\max _{s \in K}\left|L_{1}(s+i \tau)-s / P_{1}(s)\right|<\varepsilon \\
\max _{s \in K}\left|L_{2}(s+i \tau)-s_{0} / P_{2}(s)\right|<\varepsilon \\
\max _{1 \leq j \leq 2} \max _{s \in K}\left|P_{j}(s+i \tau)-P_{j}(s)\right|<\varepsilon \\
\max _{3 \leq j \leq n} \max _{s \in K}\left|L_{j}(s+i \tau)-\varepsilon\right|<\varepsilon
\end{array}\right\}>0
$$


Then easy calculation shows that

$$
\liminf _{T \rightarrow \infty} \frac{1}{T} \mu\left\{\tau \in[0, T]: \max _{s \in K}\left|L(s+i \tau)-\left(s-s_{0}\right)\right|<\varepsilon .\right\}>0 .
$$

Taking suitable $\varepsilon$ we get

$$
\liminf _{T \rightarrow \infty} \frac{1}{T} \mu\left\{\tau \in[0, T]: \max _{s \in \partial K}\left|L(s+i \tau)-\left(s-s_{0}\right)\right|<\delta=\left|s-s_{0}\right| .\right\}>0 .
$$

Hence using the classical Rouché theorem completes the proof.

Remark 1 Let us notice that Theorems 2 and 3 hold also for any functions $P_{1}, \ldots, P_{n}$, where at least two of them are absolutely convergent general Dirichlet series without zeros in $D$ and all these functions satisfy the following estimate

$$
\max _{\sigma_{1} \leq \operatorname{Re} s \leq \sigma_{2}}\left|P_{j}(s)\right| \ll 1, \quad j=1,2, \ldots, n
$$

for every $\frac{1}{2}<\sigma_{1}<\sigma_{2}<1$.

\section{Zeta functions associated to arithmetic functions}

In this section we give some examples of zeta functions associated to some arithmetic functions, for which universality property can be easily deduced from the above theorems.

Let $v_{p}(n)$ denote the largest integer value of $k$ such that $p^{k} \mid n$. Then the Liouville function, denoted by $\lambda(n)$, is defined by

$$
\lambda(n)=(-1)^{\sum_{p \mid n} v_{p}(n)} .
$$

The generating Dirichlet series for the Liouville function gives for $\sigma>1$

$$
\sum_{n=1}^{\infty} \frac{\lambda(n)}{n^{s}}=\frac{\zeta(2 s)}{\zeta(s)}
$$

Hence by Theorem 1 it is easy to see that $\zeta(2 s) / \zeta(s)$ is universal, since $1 / \zeta(s)$ is hybridly universal.

Now, let us define a function $\kappa(n)$ as the product $\prod_{p \mid n} v_{p}(n)$. Then, for the Dirichlet series associated to $\kappa(n)$ we have

$$
\sum_{n=1}^{\infty} \frac{\kappa(n)}{n^{s}}=\frac{\zeta(s) \zeta(2 s) \zeta(3 s)}{\zeta(6 s)}, \quad(\sigma>1)
$$

which defines the universal function in the strip $D$. Note that by Theorem 1 we can not obtain the universality for the zeta-functions associated to subgroups of an abelian 
group of rank at most 2 (see for example [10]), which has a similar representation given by

$$
\zeta(s)^{2} \zeta(2 s)^{2} \zeta(2 s-1)^{2} \prod_{p}\left(1+p^{-2 s}-2 p^{-3 s}\right)
$$

Analogously, we obtain universality theorems for zeta functions related to the squarefree numbers i.e. the functions defined as $\sum_{n=1}^{\infty} \frac{\mu(n)^{2}}{n^{s}}=\frac{\zeta(s)}{\zeta(2 s)}$ and $\sum_{n=1}^{\infty} \frac{2^{\omega(n)}}{n^{s}}=\frac{\zeta(s)^{2}}{\zeta(2 s)}$ for $\sigma>1$, where $\mu(\cdot)$ denotes the Möbius function and $2^{\omega(n)}$ counts the number of square-free divisors.

Generally, we can obtain the universality for various Dirichlet series connected with $\zeta(s)$ in [23, Sect. 1.2].

To obtain the joint universality theorem let us take a zeta function defined as the Dirichlet series of the divisor function twisted with non-equivalent Dirichlet characters. More precisely, for a complex number $\alpha$ we define the divisor function $\sigma_{\alpha}(n)=$ $\sum_{d \mid n} d^{\alpha}$. Then, for a Dirichlet character $\chi$, let

$$
E(s ; \chi, \alpha)=\sum_{n=1}^{\infty} \frac{\sigma_{\alpha}(n)}{n^{s}} \chi(n), \quad(\sigma>\min (1+\operatorname{Re} \alpha, 1)) .
$$

The analytic continuation of $E(s ; \chi, \alpha)$ is given by the equation (see [3, Theorem 3])

$$
E(s ; \chi, \alpha)=L(s, \chi) L(s-\alpha, \chi)=E(s-\alpha ; \chi,-\alpha) .
$$

Therefore Theorem 1 gives the joint universality theorem for $E\left(s ; \chi_{1}, \alpha\right), \ldots$, $E\left(s ; \chi_{n}, \alpha\right)$ in the strip $D$, whenever $\operatorname{Re} \alpha<-1 / 2$ and $\chi_{1}, \ldots, \chi_{n}$ are non-equivalent Dirichlet characters. Moreover, taking $\operatorname{Re} \alpha>1 / 2$ makes the functions $E(s$; $\left.\chi_{1}, \alpha\right), \ldots, E\left(s ; \chi_{n}, \alpha\right)$ jointly universal in the strip $D+\operatorname{Re} \alpha$.

To present an application of Theorem 2 we define the Estermann zeta functions as

$$
E\left(s ; \frac{k}{l}, \alpha\right)=\sum_{n=1}^{\infty} \frac{\sigma_{\alpha}(n)}{n^{s}} \exp \left(2 \pi i n \frac{k}{l}\right) \quad(\sigma>\max (1+\operatorname{Re} \alpha, 1))
$$

where $k, l$ are coprime integers. For prime $l$ (see [3, Theorem 2]) we have

$$
\begin{aligned}
& E\left(s ; \frac{k}{l}, \alpha\right)=\frac{1}{\varphi(l)} \sum_{\substack{\chi \bmod l \\
\chi \neq \chi_{0}}} \tau(\bar{\chi}) \chi(k) L(s, \chi) L(s-\alpha, \chi) \\
& +\Lambda(s ; \alpha) L\left(s, \chi_{0}\right) L\left(s-\alpha, \chi_{0}\right),
\end{aligned}
$$

where

$$
\Lambda(s ; \alpha)= \begin{cases}\frac{2 l-l^{1-s}-l^{s}}{l^{s}(l-1)\left(1-l^{-s}\right)^{2}}, & \text { if } \alpha=0 \\ \frac{l-l^{1+\alpha-s}-l^{1+2 \alpha}+l^{1+2 \alpha-s}-l^{s}+l^{\alpha+s}}{l^{s}(l-1)\left(1-l^{\alpha}\right)\left(1-l^{-s}\right)\left(1-l^{\alpha-s}\right)}, & \text { otherwise }\end{cases}
$$


Hence using the estimate $\max _{s \in D} \Lambda(s ; \alpha) \ll 1$, Remark 1 and the equation $E(s$; $\left.\frac{k}{l}, \alpha\right)=E\left(s-\alpha ; \frac{k}{l},-\alpha\right)$ leads to the following result.

Proposition 1 Suppose $\operatorname{Re} \alpha<-1 / 2$ and $l \geq 5$ is prime. Let $K \subset D$ be a compact set with connected complement, and $f(s)$ be a continuous function in $K$ which is analytic in the interior of $K$. Then, for every $\varepsilon>0$, we have

$$
\liminf _{T \rightarrow \infty} \frac{1}{T} \mu\left\{\tau \in[0, T]: \max _{s \in K}\left|E\left(s+i \tau ; \frac{k}{l}, \alpha\right)-f(s)\right|<\varepsilon\right\}>0 .
$$

Moreover, assuming $\operatorname{Re} \alpha>1 / 2$ gives the same inequality for any compact set $K \subset$ $D+\operatorname{Re} \alpha$ with connected complement.

Noteworthy is the fact that Garunkštis, Laurinčikas, Šleževičienė and Steuding [3, Theorem 4] proved the universality theorem for the Estermann zeta function (for the same parameters as in Proposition 1) in the strip $\beta<\sigma<1$ under the additional assumption that

$$
\sum_{j=1}^{\infty} \frac{\sigma_{\alpha}\left(p^{j}\right)}{p^{j / \beta}}<1
$$

holds for $p=2,3$ and some $\beta \in(1 / 2,1)$. Therefore, we see that using the hybrid universality allows to relax the above assumption and simultaneously simplifies the proof of the universal property for the Estermann zeta function.

\section{Zeta functions associated to symmetric matrices}

Zeta functions associated to the prehomogeneous vector space $V_{n}$ of $n \times n$ rational symmetric matrices are very interesting objects for several reasons. For example, their special values at non-positive integers describe the contribution of central unipotent elements to the dimensions of the Siegel modular form.

For the case $n=2$, these zeta functions have been investigated by Siegel, Morita, Shintani, Sato and Arakawa. For the case $n \geq 3$, explicit forms of the zeta functions associated to symmetric matrices have been proved by Ibukiyama and Saito in [8] and [9].

We use the same definitions and notation of [8]. For each field $F$ in $\mathbb{C}$, we put $V_{n}(F)=V_{n} \otimes_{\mathbb{Q}} F$. Then the pair $\left(G L_{n}(\mathbb{C}), V_{n}(\mathbb{C})\right)$ is a prehomogeneous vector space through the action: $V_{n}(\mathbb{C}) \in x \longmapsto g x^{t} g \in V_{n}(\mathbb{C})$ for $g \in G L_{n}(\mathbb{C})$. Denote by $V_{n}^{j}$ the subset of $V_{n}(\mathbb{R})$ consisting of matrices with $j$ positive and $n-j$ negative eigenvalues. We fix a lattice $L \subset V_{n}(\mathbb{R})$ which is invariant under $S L_{n}(\mathbb{Z})$; put $L^{(j)}:=L \cap V_{n}^{j}$ and denote by $L^{(j)} / \sim$ the set of $S L_{n}(\mathbb{Z})$ equivalence classes in $L^{(j)}$. Then, except for the case $n=2$ and $j=1$, the zeta functions $\zeta_{j}(s, L)$ of signature $(j, n-j)$ attached to $L$ are given by

$$
\zeta_{j}(s, L):=\frac{2 \prod_{k=1}^{n} \Gamma(k / 2)}{\pi^{n(n+1) / 4}} \sum_{x \in L^{(j)} / \sim} \frac{\mu(x)}{|\operatorname{det} x|^{s}}
$$


where $\mu(x)$ is defined as follows: let $G_{+}:=\left\{g \in G L_{n}(\mathbb{R}): \operatorname{det} g>0\right\}$, and

$$
d g:=(\operatorname{det} g)^{-n} \prod_{1 \leq j, k \leq n} d g_{j k}
$$

be the measure on $G_{+}$. As a measure on $V_{n}^{j}$, we take $d y:=\prod_{1 \leq j \leq k \leq n} d y_{j k}$. For $x \in L^{(j)}$, let $U$ be a relatively compact open set in $V_{n}^{j}$ and let $Y$ be the domain in $G_{+}$ which is mapped to $U$ by the mapping $x \longmapsto g x^{t} g$. Let $\Gamma_{x}$ be the stabilizer of $x$ in $S L_{n}(\mathbb{Z})$, and $Y_{0}$ a fundamental domain with respect to the right action of $\Gamma_{x}$. The ratio

$$
\mu(x):=\int_{Y_{0}} d g / \int_{U}|y|^{-(n+1) / 2} d y
$$

is finite and independent of the choice of $U$ unless $n=2$ and $j=1$. This completes the definition of $\mu(x)$ and $\zeta_{j}(s, L)$.

For a ring $R$, we denote by $S_{n}(R)$ the set of all symmetric matrices with coefficients in $R$ and by $S_{n}(R)_{e}$ its subset consisting of even elements, that is, the elements whose diagonal elements are contained in $2 R$. In particular, $S_{n}(\mathbb{Q})=V_{n}(\mathbb{Q})$. According to [8, Lemma 1.1], for $S L_{n}(\mathbb{Z})$ invariant lattices in $S_{n}(\mathbb{Q})$, we have the following. Let $n \geq 3$ and let

$$
L_{n}:=S_{n}(\mathbb{Z}), \quad L_{n}^{*}:=\frac{1}{2} S_{n}(\mathbb{Z})_{e}
$$

If a lattice $L$ in $V_{n}(\mathbb{Q})$ is invariant under $S L_{n}(\mathbb{Z})$, then up to a nonzero element of $\mathbb{Q}^{\times}$, $L$ coincides with $L_{n}$ or $L_{n}^{*}$.

Thus we may assume that $L=L_{n}$ or $L_{n}^{*}$. It should be noted that $\zeta_{j}(s, L)$ depends only on the determinant $\eta:=(-1)^{n-j}$ and the Hasse invariant $\theta:=$ $(-1)^{(n-j)(n-j+1) / 2}$ of $x \in V_{n}^{j}$ (see [8, Sect. 2]). Hence we denote

$$
\zeta_{\eta, \theta}(s, L):=\zeta_{j}(s, L)
$$

for $L_{n}$, or $L_{n}^{*}$. The following theorem is proved by Ibukiyama and Saito [8]. For the sake of simplicity, we put

$$
\begin{aligned}
& b_{n}(s ; L)= \begin{cases}\frac{\left|\prod_{k=1}^{[n / 2]} B_{2 k}\right|}{2^{n-1}\left(\frac{n-1}{2}\right) !} & \text { if } L=L_{n}, \\
\frac{\prod_{k=1}^{[n / 2]} B_{2 k} \mid}{2^{n-1}\left(\frac{n-1}{2}\right) !} 2^{(n-1) s} & \text { if } L=L_{n}^{*}\end{cases} \\
& Q_{n}(s)=\theta \eta^{(n+1) / 2}(-1)^{\left(n^{2}-1\right) / 8} \zeta(s) \prod_{k=1}^{[n / 2]} \zeta(2 s-2 k) \\
& P_{n}(s ; L)= \begin{cases}2^{(n-1) / 2} \prod_{k=1}^{[n / 2]} \zeta(2 s-(2 k-1)) & \text { if } L=L_{n} \\
\prod_{k=1}^{[n / 2]} \zeta(2 s-(2 k-1)) & \text { if } L=L_{n}^{*}\end{cases}
\end{aligned}
$$


Theorem B ([8, Theorem 1.2]) Let $n \geq 3$ be an odd positive integer. Then we have

$$
\zeta_{\eta, \theta}(s, L)=b_{n}(s ; L)\left(P_{n}(s ; L) \zeta(s-(n-1) / 2)+Q_{n}(s)\right)
$$

It was also showed in [8, Theorem 1.3] that when $n$ is even, these zeta functions consist of two parts; one part is a product of shifted Riemann zeta functions and the other part is composed of shifted Riemann zeta functions and the Dirichlet series attached to an Eisenstein series of half integral weight of one variable.

Moreover, Ibukiyama and Saito [9] gave an explicit form for $L$-functions associated to the space of $n \times n$ symmetric matrices and to Dirichlet characters. These $L$-functions consist of two terms. Each term is essentially a product of Dirichlet $L$-functions except that if $n$ is even, a certain Dirichlet series appears in the product constituting one term.

Now we show the universality theorem for zeta functions associated to symmetric matrices $\zeta_{\eta, \theta}\left(s, L_{n}\right)$ and $\zeta_{\eta, \theta}\left(s, L_{n}^{*}\right)$. Put $D_{n}:=\{s \in \mathbb{C}: n / 2<\operatorname{Re}(s)<(n+1) / 2\}$.

Theorem 4 Assume that $L=L_{n}$ or $L_{n}^{*}$ and $n \geq 3$ is an odd positive integer. Let $K \subset D_{n}$ be a compact set with connected complement. Moreover, let $f(s)$ be a function continuous on $K$ and analytic in the interior of $K$, such that $f(s) \neq b_{n}(s ; L) Q_{n}(s)$ for all $s \in K$. Then, for every $\varepsilon>0$, we have

$$
\liminf _{T \rightarrow \infty} \frac{1}{T} \mu\left\{\tau \in[0, T]: \max _{s \in K}\left|\zeta_{\eta, \theta}(s+i \tau, L)-f(s)\right|<\varepsilon\right\}>0 .
$$

Proof Notice that the functions $b_{n}(s ; L), P_{n}(s ; L)$ and $Q_{n}(s)$ are an absolute convergent general Dirichlet series without zeros in the strip $D_{n}$ for an odd $n \geq 3$. Hence, using Theorem 1 completes the proof.

\section{Double zeta functions}

In this section, we consider the universality of Euler-Zagier double $L$-functions and Euler-Zagier-Hurwitz type of double zeta functions defined by the absolute convergent series

$$
L_{2}\left(s_{1}, s_{2} ; \chi_{1}, \chi_{2}\right):=\sum_{n_{1}>n_{2}>0} \frac{\chi_{1}\left(n_{1}\right) \chi_{2}\left(n_{2}\right)}{n_{1}^{s_{1}} n_{2}^{s_{2}}}
$$

and

$$
\zeta_{2}\left(s_{1}, s_{2} ; \alpha_{1}, \alpha_{2}\right):=\sum_{n_{1}>n_{2} \geq 0} \frac{1}{\left(n_{1}+\alpha_{1}\right)^{s_{1}}\left(n_{2}+\alpha_{2}\right)^{s_{2}}}, \quad \text { respectively, }
$$

where $0<\alpha_{1}<\alpha_{2} \leq 1$ and $\operatorname{Re}\left(s_{1}\right)>1, \operatorname{Re}\left(s_{2}\right) \geq 1$. The function $\zeta_{2}\left(s_{1}, s_{2} ; \alpha_{1}, \alpha_{2}\right)$ is a generalization of the Hurwitz zeta-function $\zeta(s, \alpha):=\sum_{n=0}^{\infty}(n+\alpha)^{-s}$ and can be 
continued meromorphically $\mathbb{C}^{2}$ with possible singularities at: $s_{1}=1, s_{1}+s_{2} \in \mathbb{Z}_{\leq 2}$ (see [1, Theorems 1 and 2]).

Noteworthly is the fact that the meromorphic continuation of these functions is a simple consequence of the meromorphic continuation of the multiple zeta function defined in the 2-dimensional case as $\zeta\left(s_{1}, s_{2} ; 1,1\right)$ (see [14]).

In addition, Matsumoto showed a functional equation for the Euler-Zagier-Hurwitz type of double zeta functions in [13] and the first author showed the universality for the Euler-Zagier-Hurwitz type of multiple zeta functions in [16, Theorem 2.1]. Note that the Hurwitz zeta-function $\zeta(s, \alpha)$ is non-vanishing if $\sigma \geq 1+\alpha$ (see [11, Theorem 8.1.1]).

Theorem C (see [16, Theorem 2.1]) Fix $0<\alpha_{2} \leq 1$ and $\operatorname{Re}\left(s_{2}\right)>3 / 2$. Let $0<$ $\alpha_{1}<1$ be transcendental and $f\left(s_{1}\right)$ be a function analytic in the interior of $K$ and continuous on $K$. If $\zeta\left(s_{2}, \alpha_{2}\right) \neq 0$, then for every $\varepsilon>0$,

$$
\liminf _{T \rightarrow \infty} \frac{1}{T} \mu\left\{\tau \in[0, T]: \max _{s_{1} \in K}\left|\zeta_{2}\left(s_{1}+i \tau, s_{2} ; \alpha_{1}, \alpha_{2}\right)-f\left(s_{1}\right)\right|<\varepsilon\right\}>0 .
$$

Our goal is to apply Theorem 1 to obtain universality for functions introduced above. In fact, it is sufficient to observe that

$$
\begin{aligned}
2 \zeta_{2}(s, s ; \alpha, \alpha) & =\zeta(s, \alpha)^{2}-\zeta(2 s, \alpha), \\
2 L_{2}(s, s ; \chi, \chi) & =L(s, \chi)^{2}-L\left(2 s, \chi^{2}\right) .
\end{aligned}
$$

Therefore, using the fact that Dirichlet L-functions and Hurwitz zeta functions with transcendental parameter are hybridly universal we get the following theorems.

Theorem 5 Suppose that $\chi_{1}, \ldots, \chi_{m}$ are pairwise non-equivalent Dirichlet characters and $K_{j} \subset D$ are compact sets with connected complement. Moreover, assume that $f_{j}(s)$ for $1 \leq j \leq m$ are functions analytic in $K_{j}$ and analytic in the interior of $K_{j}$ such that $2 f_{j}(s) \neq-L\left(2 s, \chi_{j}^{2}\right)$ for all $s \in K_{j}$. Then, for every $\varepsilon>0$, we have

$$
\liminf _{T \rightarrow \infty} \frac{1}{T} \mu\left\{\tau \in[0, T]: \max _{1 \leq j \leq m} \max _{s \in K_{j}}\left|L_{2}\left(s+i \tau, s+i \tau ; \chi_{j}, \chi_{j}\right)-f_{j}(s)\right|<\varepsilon\right\}>0 .
$$

Theorem 6 Assume that $K \subset D$ is a compact set with connected complement. Let $0<\alpha \leq 1$ be a transcendental real number and $f(s)$ be a function analytic in the interior $K$ and continuous on $K$ such that $2 f(s) \neq-\zeta(2 s, \alpha)$ for all $s \in K$. Then, for every $\varepsilon>0$, we have

$$
\liminf _{T \rightarrow \infty} \frac{1}{T} \mu\left\{\tau \in[0, T]: \max _{s \in K}\left|\zeta_{2}(s+i \tau, s+i \tau ; \alpha, \alpha)-f(s)\right|<\varepsilon\right\}>0 .
$$

Remark 2 In fact, instead of the assumption that $2 f_{j}(s) \neq-L\left(2 s, \chi_{j}^{2}\right)$ in Theorem 5.1 (resp. $2 f(s) \neq-\zeta(2 s, \alpha)$ in Theorem 5.2) it suffices to suppose that there exists the square-root functions of $2 f_{j}(s)+L\left(2 s, \chi_{j}^{2}\right)(\operatorname{resp} .2 f(s)+\zeta(2 s, \alpha))$. 
Remark 3 Following the proof of the above theorem we can obtain the universality for multiple Hurwitz zeta functions and multiple L-functions as well. In this case we need the more technical assumption on function $f(s)$. For example, for triple Hurwitz zeta functions we have

$$
\zeta_{3}(s, s, s ; \alpha, \alpha, \alpha)=\frac{1}{6} \zeta(s, \alpha)^{3}-\frac{1}{2} \zeta(s, \alpha) \zeta(2 s, \alpha)+\frac{1}{3} \zeta(3 s, \alpha) .
$$

Hence the analogous of Theorem 5.2 needs the assumption that the approximated function can be expressed as $1 / 6 f(s)^{3}-1 / 2 f(s) \zeta(2 s, \alpha)+1 / 3 \zeta(3 s, \alpha)$ for suitable function $f(s)$ continuous on $K$ and analytic in the interior of $K$.

Acknowledgments We would like to thank the anonymous referee for very useful comments and remarks.

Open Access This article is distributed under the terms of the Creative Commons Attribution Noncommercial License which permits any noncommercial use, distribution, and reproduction in any medium, provided the original author(s) and source are credited.

\section{References}

1. Akiyama, S., Ishikawa, H.: On analytic continuation of multiple $L$-functions and related zeta-functions. Analytic number theory (Beijing/Kyoto, 1999), Dev. Math., 6, pp. 1-16, Kluwer Acad. Publ., Dordrecht (2002)

2. Bagchi, B.: The statistical behaviour and universality properties of the Riemann Zeta-function and other allied dirichlet series. Ph.D. thesis, Calcutta, Indian Statistical Institute (1981)

3. Garunkštis, R., Laurinčikas, A., Šleževičienè, R., Steuding, J.: On the universality of the Estermann zeta-function. Analysis 22, 285-296 (2002)

4. Gonek, S.M.: Analytic properties of Zeta and L-functions. Ph.D. thesis, Universality of Michigan (1979)

5. Javtokas, A., Laurinčikas, A.: A joint universality theorem for periodic Hurwitz zeta-functions. Bull. Aust. Math. Soc 78, 13-33 (2008)

6. Laurinčikas, A., Skerstonaitả, S.: A joint universality theorem for periodic Hurwitz zeta-functions II. Lith. Math. J. 49(3), 287-296 (2009)

7. Kaczorowski, J., Kulas, M.: On the non-trivial zeros off line for L-functions from extended Selberg class. Monatshefte Math. 150, 217-232 (2007)

8. Ibukiyama, T., Saito, H.: On zeta functions associated to symmetric matrices, I. An explicit form of zeta functions. Amer. J. Math. 117, 1097-1155 (1995)

9. Ibukiyama, T., Saito, H.: On zeta functions associated to symmetric matrices. III. An explicit form of L-functions. Nagoya Math. J. 146, 149-183 (1997)

10. Laurinčikas, A.: The universality of Dirichlet series attached to finite abelian groups, Number theory (Turku, 1999). pp. 179-211. de Gruyter, Berlin (2001)

11. Laurinčikas, A., Garunkšitis, R.: The Lerch zeta-function. Kluwer Academic Publishers, Dordrecht (2002)

12. Laurinčikas, A., Matsumoto, K.: Joint value-distribution theorems for the Lerch zeta-functions III. In: "Analytic and probabilistic methods in number theory", Proceedings of the 4th International Conference in Honour of J. Kubilius, A. Laurinčikas, E. Manstavičius (eds.), pp. 87-98. TEV, Vilnius (2007)

13. Matsumoto, K.: Functional equations for double zeta-functions. Math. Proc. Cambridge Philos. Soc 136(1), 1-7 (2004)

14. Ram Murty, M., Sinha, K.: Multiple Hurwitz zeta functions. Multiple Dirichlet Series, Automorphic Forms, and Analytic Number Theory, Proceedings of Symposia in Pure Mathematics 75, 135156 (2006)

15. Nakamura, T.: The existence and the non-existence of joint t-universality for Lerch zeta functions. J. Number Theory 125(2), 424-441 (2007) 
16. Nakamura, T.: Zeros and the universality for the Euler-Zagier Hurwitz type of multiple zeta-functions. Bull. Lond. Math. Soc 41, 691-700 (2009)

17. Pańkowski, Ł.: Hybrid joint universality theorem for the Dirichlet L-functions. Acta Arith 141(1), 59$72(2010)$

18. Pańkowski Ł.: Hybrid joint universality theorem for L-functions without the Euler product (submitted)

19. Sander, J., Steuding, J.: Joint universality for sums and products of Dirichlet L-functions. Analysis 26, 295-312 (2006)

20. Steuding, J.: Value-Distribution of L-functions. Springer, Berlin (2007)

21. Voronin S.M.: Theorem on the universality of the Riemann zeta function. Izv Akad Nauk SSSR Ser. Mat. 39:475-486 (1975, in Russian)

22. Voronin, S.M.: Theorem on the universality of the Riemann zeta function. Math. USSR Izv 9, 443453 (1975)

23. Titchmarsh, E.C.: In: Heath-Brown D. R. (ed.) The theory of the Riemann zeta-function. 2nd edn. The Clarendon Press, Oxford University Press, New York (1986) 